\title{
The role of laparoscopy in evaluating cytoreduction in advanced ovarian cancer
}

\author{
Interview with Anna Fagotti ${ }^{1}$, Giovanni Scambia ${ }^{1}$ and Sandro Pignata $^{2}$, Carmela Pisano ${ }^{2}$ \\ by Domenica Lorusso ${ }^{3}$
}

First perspective:

Anna Fagotti ${ }^{1}$, Giovanni Scambia ${ }^{1}$

Second perspective:

Sandro Pignata ${ }^{2}$, Carmela Pisano²

\section{Introduction}

Primary cytoreductive surgery with the aim of complete cytoreduction (residual tumor $=0$ ) followed by platinum based chemotherapy represents the standard of care for advanced ovarian cancer. Nevertheless, up to $25 \%$ of advanced stage patients in the referral centers are not able to receive complete cytoreduction due to the amount of disease or to personal clinical conditions that prevent them receiving aggressive surgical interventions. A large part of clinical research in the last years has aimed at identifying a radiological (computed tomography scan) or clinical (albumin level) or serological (carcinoembryonic antigen 125 value) parameter able to predict which patients are not suitable for primary surgery and should be candidates for neoadjuvant chemotherapy. Unfortunately, none of these tools are reported to be sufficiently sensitive in identifying this population.

In recent years, minimum invasive surgery has become a new standard approach in several gynecological malignancies, and several research groups have published on the possibility of its use, before laparotomy, to evaluate the tumor spread and to predict cytoreduction. They sug-

\footnotetext{
${ }^{1}$ Gynecologic Oncology, Policlinico A. Gemelli Foundation, Università Cattolica del Sacro Cuore, Roma, Italy.

${ }^{2}$ Medical Oncology, Department of Urology and Gynecology, Istituto Nazionale Tumori Fondazione G. Pascale IRCCS, Napoli, Italy. ${ }^{3}$ Gynecologic Oncology Unit, Fondazione IRCCS, Istituto Nazionale dei Tumori, Milano, Italy.

Correspondence to:

Domenica Lorusso, MD,

Gynaecologic Oncology Unit, Fondazione IRCCS,

Istituto Nazionale dei Tumori,

Via Venezian 1, 20133 Milano, Italy.

Phone: +39 0223903697 - Fax: +3902 23902349

E-mail: domenica.lorusso@istitutotumori.mi.it

CANCER BREAKING NEWS 2017;5(2):18-21

DOI: $10.19156 / \mathrm{cbn} .2017 .0044$
}

gest that patients with a predictive laparoscopic score superior to 8 or 10 should receive neoadjuvant chemotherapy, because the possibility of achieving optimal cytoreduction in these patients is less than $10 \%$ and by using laparoscopy (LPS), patients are spared unnecessary laparotomies. On the contrary, other referral centers refuse it, because at least $80 \%$ of ovarian cancer patients have a predictive score $>8$ and as such should be candidates for neoadjuvant chemotherapy, with a significant worsening of the patient's prognosis. Furthermore, centers using routine LPS report a percentage of cytoreduction significantly lower with respect to centers using laparotomy to assess cytoreduction and that LPS has the limitation of not carefully evaluating mesenteric infiltration, which is the principal reason for non-cytoreductibility or retroperitoneal disease.

In this context, we asked four experts in the field of gynecologic oncology surgery to share their experience and opinions on this hot topic. Anna Fagotti and Giovanni Scambia, from the Policlinico A. Gemelli Foundation in Rome, and Sandro Pignata and Carmela Pisano, from the Istituto Nazionale Tumori Fondazione G. Pascale IRCCS in Naples will present their perspectives on the use of LPS in the prediction of ovarian cancer cytoreductibility.

\section{Do you consider laparoscopy (LPS) as an appropriate tool to predict which advanced ovarian cancer patients are able to receive complete cytoreduction? Why/Why not?}

\section{First perspective}

The most frequent reason for not achieving complete cytoreduction in women with advanced ovarian cancer is the presence of small bowel carcinomatosis (up to $80 \%$ of the cases) [1,2]. Multiple models encompassing serologic markers and imaging modalities have been proposed as a means to preoperatively estimate the likelihood of no gross residual tumor (NGR) at primary deb- 
ulking surgery (PDS), with disappointing results. Our recent data from staging laparoscopy (S-LPS) demonstrate that the model is very accurate when NGR is estimated, but it may fail in predicting optimal resection when the residual tumor is parenchymal and/or retroperitoneal. However, it is very useful to assess small bowel carcinomatosis, with higher accuracy than any other imaging technique [2].

\section{Second perspective}

I believe LPS is the main attempt undertaken, and published in the literature, in the search of a tool able to select patients for optimal cytoreduction able to avoiding non-useful interventions that can delay the start of chemotherapy. Data show that LPS is reliable and offers the mandatory need of a histological biopsy before starting neoadjuvant therapy in the inoperable cases. The knowledge of the biology and histology of the tumor must be considered mandatory in the modern age of ovarian cancer therapy. Data have shown that LPS results are reproducible even in centers with less experience with the technique, as clearly shown by the MITO 13-Olympia study [3].

\section{What in your view are the limits of laparoscopic evaluation?}

\section{First perspective}

The only issue S-LPS can address in women with advanced epithelial ovarian cancer (AEOC) is extent and localization of intra-peritoneal disease. Thus, any other extra-abdominal localization as well as abdominal intraparenchymal and retroperitoneal metastases cannot be assessed. The value of this approach consists in the fact that a) most AEOC cases have trans-celomatic diffusion, which is the key point for surgical debulking and finally prognosis, b) standard imaging techniques have a high false negative rate in term of intraperitoneal staging [4].

\section{Second perspective}

LPS is a surgical intervention that requires an organization that make possible to convert to open laparotomy immediately operability is found at the laparoscopic assessment. In terms of organization of the daily practice this is not always possible, particularly because an open laparotomic cytoreduction requires several hours in the surgical theatre. Not all centers have this possibility and, in these centers, LPS may cause a significant delay to the start of therapy.

\section{Do you consider the usefulness of LPS is the same in all the centers regardless of}

\section{the percentage of cytoreduction reached in the center?}

\section{First perspective}

This question requires two answers. a) If you consider centers with high rates of cytoreduction, you may argue it is an expensive and time-consuming procedure with low benefits. However, it is quite common that the intraabdominal surgical field is different from the one reported from imaging, especially if radiological tests are performed in external hospitals, with no revision from a dedicated radiologist. Moreover, about $8-10 \%$ of supposed $\mathrm{AEOC}$ are in fact metastatic gastrointestinal or breast tumours. In our opinion, S-LPS represents an easy approach to verify histology and intra-peritoneal diffusion of disease, before any therapeutic decision, to avoid unnecessary laparotomies and their related side effects, even in a small proportion of women. b) If you consider centers with low rate of cytoreduction, you may argue that laparoscopy is used inappropriately. If this is the case, I do not think the problem is whether one patient is considered resectable or unresectable. If a center does not have the skills and/or resources and/or motivation towards PDS, thus leading to a low rate of PDS, physicians can use any approach to support their clinical decision, including laparotomy. However, I would suggest being cautious in defining high/low rate of cytoreduction between centers. Indeed, this rate is strongly dependent on which is the denominator, in term of absolute number and type of patients received.

\section{Second perspective}

There are centers where residual zero is obtained in more than $50 \%$ of the cases, with residual less than $1 \mathrm{~cm}$ in another $30-40 \%$ of cases. In these centers, there is a need to perform 10 LPSs to select only one patient for neoadjuvant chemotherapy. In these centers the usefulness of LPS is reduced. However, the question of centralization of this difficult surgery in high volume centers is an issue in several countries and, overall, the proportion of patients operated on in these highly specialized centers is still limited.

\section{In a dynamic process such as surgery, in which all the referral centers involved in ovarian cancer treatment globally publish their learning curve on surgical expertise, and declare that they are actually able to cytoreduce patients that only few years ago received neoadjuvant chemotherapy because they were not considered}


cytoreductible, is it possible that a predetermined cut-off (Fagotti score 8) could represent a blocking process in the surgical learning curve and, as such, an obstacle in the dynamic process of amelioration of surgical techniques?

\section{First perspective}

This is exactly the same question we tried to answer with the paper from Petrillo et al, in 2015 [2]. In fact, the laparoscopic-predictive index (LPS-PI) was initially designed, and prospectively validated, before the achievement of relevant improvements in the surgical management of AEOC, which have significantly increased the chance of achieving a complete PDS, with significant survival benefit. For these reasons, we retrospectively analysed our large single Institution series of AEOC patients, who received S-LPS followed by a maximal laparotomic surgical effort, after the introduction of upper abdominal surgery, with the aim of developing an updated LPS-PI able to predict the chance of complete PDS nowadays. We demonstrated that the introduction of upper abdominal procedures to the surgical skills of gynecologic oncologists has modified the cut-off value of laparoscopic score from 8 up to 10 , increasing the chances to achieve complete PDS and reducing the rate of unnecessary laparotomy from $40.5 \%$ to $33.2 \%$. This is in line with other previously reported series, and with any other score or cut-off value used in clinical practice. Indeed, scoring systems are successfully employed in several fields of medicine in order to assist physicians to make appropriate complex therapeutic decisions, but they need to be continuously updated to keep pace with therapeutic innovations, and technical advancements introduced into clinical practice.

\section{Second perspective}

Personally, I do not believe this. Most surgeons are convinced of the role of primary cytoreduction. Also, the trials of neoadjuvant therapy showing equal outcome compared with primary surgery suggest that primary cytoreduction is the standard of care. The TRUST trial (ClinicalTrials.gov Identifier: NCT02828618), making the same comparison, but only in certified centers able to perform maximal cytoreduction, will definitively answer the question of the role of neoadjuvant chemotherapy.

\section{Referral centers who evaluate} cytoreduction by LPS publish percentages of cytoreductions that are usually lower with respect to centers who do not use

LPS. What is your explanation for this?

\section{First perspective}

I am not sure that this is correct. If you look at the recent series published from popular centers for surgical treatment of AEOC, not using S-LPS, the rate of complete cytoreduction ranges between $35.8 \%$ [5], 51\% [6] and 51\% [1] when considering their entire population of primary AEOC. In our recent publications, we reported a rate of complete cytoreduction ranging from $45.5 \%$ [7], to $57.7 \%$ [2] and $90 \%$ [8].

\section{Second perspective}

The possibility that, in some centers, LPS may cause an increase of patients that undergo neoadjuvant therapy and a decrease of optimally cytoreduced patients at primary surgery is real. However, this was not the intent of those who designed and studied the laparoscopic score that, in my mind, remains the most objective assessment of the level of operability of patients. As with every tool, even LPS must be used in the proper hands and in the interest of the patients.

\section{A recent publication suggests that the} time when the surgical operation is undertaken correlates with the outcome of surgery in terms of percentage of optimal cytoreduction, thus confirming to the scientific community that the possibility of obtaining optimal cytoreduction is not only a problem of surgical expertise (which remains the most important factor), but it is also influenced by the psychological attitude of the surgeon. Do you believe, in such a context, that the laparoscopic approach may provide a further excuse to unwilling or unmotivated surgeons to give up 4 to 5 hours of surgery?

\section{First perspective}

We all agree that any diagnostic/therapeutic procedure in ovarian cancer patients should be centralized in referral centers. A referral center should be considered if an adequate percentage of cytoreduction is obtained, based on international guidelines, but this value can be greatly influenced by several biases, as previously explained. Thus, in a so-called referral center, any procedure can be used in the interest of the patient, with no risk of providing any psychological excuse to the surgeon. However, I 
would suggest performing this long surgery early in the morning or changing surgical staff after 6 hours.

\section{Second perspective}

In an ideal world, all patients with ovarian cancer should be operated on in referral centers. These centers have enough organization to make timely interventions possible. The psychological attitude of the surgeon is important, but I see a future where more centers are certified and outcomes are verified. In the future, there will be less room for personal attitudes and more space for comparison among different centers. I also believe that the extraordinary task of the present is to prepare the

\section{References}

1. Heitz F, Harter P, Alesina PF et al. Pattern of and reason for postoperative residual disease in patients with advanced ovarian cancer following upfront radical debulking surgery. Gynecol Oncol 2016;141(2):264-70.

2. Petrillo M, Vizzielli G, Fanfani F et al. Definition of a dynamic laparoscopic model for the prediction of incomplete cytoreduction in advanced epithelial ovarian cancer: proof of a concept. Gynecol Oncol 2015;139(1):5-9.

3. Fagotti A, Vizzielli G, De Iaco P et al. A multicentric trial (Olympia-MITO 13) on the accuracy of laparoscopy to assess peritoneal spread in ovarian cancer. Am J Obstet Gynecol 2013;209(5):462 e1- e11.

4. Michielsen K, Vergote I, Op de Beeck K et al. Whole-body MRI with diffusion-weighted sequence for staging of patients with suspected ovarian cancer: a clinical feasibility study in comparison to CT and FDG-PET/CT. Eur Radiol 2014;24(4):889-901.

5. Sioulas VD, Schiavone MB, Kadouri D et al. Optimal pri- young surgeons for primary cytoreduction, a surgery that requires good teachers and courageous new generations.

\section{Acknowledgments}

The Authors thank Ray Hill, an independent medical writer, who provided native English editing and journal styling on behalf of HPS. This editorial assistance was funded by PharmaMar, Spain.

\section{Conflicts of Interest}

The Authors declare there are no conflicts of interest in relation to this article.

mary management of bulky stage IIIC ovarian, fallopian tube and peritoneal carcinoma: Are the only options complete gross resection at primary debulking surgery or neoadjuvant chemotherapy? Gynecol Oncol 2017;145(1):15-20.

6. Mueller JJ, Zhou QC, Iasonos A et al. Neoadjuvant chemotherapy and primary debulking surgery utilization for advanced-stage ovarian cancer at a comprehensive cancer center. Gynecol Oncol 2016;140(3):436-42.

7. Fagotti A, Ferrandina G, Vizzielli G et al. Phase III randomised clinical trial comparing primary surgery versus neoadjuvant chemotherapy in advanced epithelial ovarian cancer with high tumour load (SCORPION trial): Final analysis of peri-operative outcome. Eur J Cancer 2016; 59:22-33.

8. Petrillo M, Marchetti C, De Leo R et al. BRCA mutational status, initial disease presentation, and clinical outcome in high-grade serous advanced ovarian cancer: a multicenter study. Am J Obstet Gynecol 2017. 